\title{
Variability in Interbeat Duration Influences Myocardial Contractility in Rat Cardiac Trabeculae
}

\author{
Carlos A. A. Torres ${ }^{1,2}$, Kenneth D. Varian ${ }^{1}$ and Paul M. L. Janssen ${ }^{1, *}$ \\ ${ }^{1}$ Department of Physiology and Cell Biology, and ${ }^{2}$ Department of Emergency Medicine 304 Hamilton Hall, 1645 Neil \\ Avenue, The Ohio State University, Columbus, OH, USA
}

\begin{abstract}
There is an intense search for positive inotropic strategies. It is well known that the interbeat duration is a critical determinant of cardiac contractility. Generally, when frequency increases, so does contractile strength. We hypothesize that the beat-to-beat variability at a given heart rate also modulates cardiac contractility. To test this hypothesis, thin, uniform rat cardiac trabeculae were isolated from the right ventricle and stimulated to isometrically contract, alternating between fixed steady state versus variable inter-beat intervals (same total number of beats in each period). Trabeculae were stimulated at $4 \mathrm{~Hz}$ with interbeat variation between 20 and $120 \%(\mathrm{n}=17)$. In a second series of experiments trabeculae were stimulated at 3 different physiologic frequencies with a $40 \%$ interbeat variation. Fixed rate response was measured before and after each variable period and average force was calculated. In order to investigate the mechanism underlying the changes in contractility we used iontophoretically loaded bis-fura-2 salt to monitor intracellular calcium transients. We observed no significant change in force at $4 \mathrm{~Hz}(\mathrm{n}=17)$, and $6 \mathrm{~Hz}(\mathrm{n}=6)$ between fixed and variable pacing but observed a significant, $10 \%$ increase in contractile strength at $8 \mathrm{~Hz}$ (from 15.1 to $16.5 \mathrm{mN} / \mathrm{mm}^{2}, \mathrm{p}<0.05, \mathrm{n}=6$ ). Our results show that under certain conditions, by simply introducing variation in the beat-to-beat duration without affecting the number of beats per minute, a positive inotropic effect with corresponding changes in the calcium transients can be generated.
\end{abstract}

\section{INTRODUCTION}

The epidemic proportions that heart failure has reached in western society has prompted an intense search for positive inotropic therapies. Current approaches vary from pharmacologic to mechanical to genetic manipulation. We sought to investigate if manipulating the R-R interval within physiologic range may be a potential inotropic strategy. The influence of the duration between successive cardiac beats has long been known to be a strong determinant of contractility. Force-frequency relationships have been investigated at the level of the whole heart [1], as well as isolated tissue [2], and isolated cell level, in various species. These previous studies clearly indicate that within the physiological range, a decrease in inter-beat duration (i.e. increase in heart rate), results in an increase in contractility. However, this forcefrequency relationship only holds true for the steady-state that develops over many beats. Contractile strength on a beat-to-beat basis shows an opposite effect: the first beat(s) after switching to a higher stimulation rate are in fact weaker [3]. An instantaneous switch from a steady inter-beatduration to a shorter inter-beat period decreases force of contraction, while an increase in inter-beat period increases contraction, the latter is called post-rest potentiation [4]. In vivo, a small variation in inter-beat duration is normally observed, (beat-to-beat variability). Since force-frequency behavior and post-rest potentiation behavior are non-linear in the time domain, a given shortening of inter-beat duration does not necessarily cause an equal and opposite change in cardiac

*Address correspondence to this author at the Department of Physiology and Cell Biology, 304 Hamilton Hall, 1645 Neil Avenue, The Ohio State University, Columbus, OH, USA; Tel: +1-614-247-7838; Fax: +1-614-2924888; E-mail: janssen.10@osu.edu contractility compared to an identical lengthening of interbeat duration. In addition, this non-linearity of the inter-beat interval to force relationship depends critically on the baseline inter-beat duration (average heart rate). We thus postulate that a variable timing component superimposed on an average constant pacing rate does therefore not necessarily result in the same contractility observed at a fixed (nonvariable) identical pacing rate. In other words, average contractile force may differ depending on presence and magnitude of beat-to-beat variability; If longer beats increase the force development by a greater magnitude than an identical shortening can cause a decrease in force development then it follows that the average force development in the variable period may exceed the one observed at the fixed rate period (both with identical number of beats) and over the same amount of time.

To test our hypothesis that interbeat variability can actively contribute to cardiac contractility, for proof-ofprinciple, we isolated multicellular cardiac trabeculae, stimulated them isometrically at fixed intervals, intercalated with protocols in which a variable inter-beat duration was imposed with identical average interval and total number of beats as the fixed rate. Our results indicate that under certain conditions that fall well within the physiological range beatto-beat variability plays a role as a determinant of myocardial contractility.

\section{MATERIALS AND METHODOLOGY}

Male Rats (LBN-f1 175-225 g) were anesthetized by intraperitoneal injection of sodium pentobarbital $(60 \mathrm{mg} / \mathrm{kg})$. The investigation conforms with the Guide for the Care and Use of Laboratory Animals published by the US National Institutes of Health (NIH Publication No. 85-23, revised 
1996). After a bilateral thoracotomy, the hearts were heparinized by introducing a 25 gauge needle via the apex while still beating. Hearts were rapidly excised and placed in Krebs-Henseleit buffer containing: $120 \mathrm{mM} \mathrm{NaCl}, 5 \mathrm{mM}$ $\mathrm{KCl}, 2 \mathrm{mM} \mathrm{MgSO}_{4}, 1 \mathrm{mM} \mathrm{NaH}_{2} \mathrm{PO}_{4}, 20 \mathrm{mM} \mathrm{NaHCO}, 0.25$ $\mathrm{mM} \mathrm{Ca}{ }^{2+}$, and $10 \mathrm{mM}$ glucose in continuous equilibrium with $95 \% \mathrm{O}_{2} / 5 \% \mathrm{CO}_{2}$ resulting in a $\mathrm{pH}$ of 7.4. BDM (2,3butanedionemonoxime) $20 \mathrm{mM}$ was added to the dissection buffer to prevent cutting injury [5]. Thin (average diameter $<150 \mu \mathrm{m}$, to avoid core-hypoxia) [6, 7], uniform, nonbranched, trabeculae $(n=19)$ along the tricuspid valve were carefully dissected, their dimensions measured, and then mounted in the experimental set-up [8]. Trabeculae were perfused with the same buffer as above (without BDM) at 37 ${ }^{\circ} \mathrm{C}, 1.5 \mathrm{mM} \mathrm{Ca}^{2+}$, and allowed to stabilize for at least $30 \mathrm{~min}$ at $4 \mathrm{~Hz}$. During stabilization, optimal muscle length was obtained by stretching muscles to where small increases in length resulted in about equal increases in resting tension and active developed tension. This fixed optimal preload is equivalent to that verified at the end diastolic volume of the in vivo situation (sarcomere length of $\sim 2.2 \mu \mathrm{m}$ ) [9].

In the first group $(n=17)$, after 20 minutes of baseline stimulation at a fixed base frequency of $4 \mathrm{~Hz}$, the trabeculae were stimulated for 2-minute periods alternating between a fixed steady state period $(4 \mathrm{~Hz})$ and a variable component period (10-120\% of cycle length). Beat-to-beat variability was introduced by random stimuli intervals with algorithms such that over an identical time period there was an equal number of beats compared to the steady state. Each period of variation was both preceded and followed by a steady state period in order to help estimate general rundown (which is unavoidable in these preparations). In order to avoid computing the effects of post rest potentiation that might occur at the exact moment the protocols are switched from steady state to variable, the 2 initial beats were not taken into account during the calculations. For this proof-of-principle investigation, beat-to-beat variability was distributed linearly, and expressed as a percentage of the variation between the longest and shortest beat (e.g. at $40 \%$ variation at $4 \mathrm{~Hz}$ (R-R interval of $250 \mathrm{~ms}$ ), the inter-beat duration varied between 200 and $300 \mathrm{~ms}$ ). In the second group of experiments, trabeculae were stimulated at $4 \mathrm{~Hz}(\mathrm{n}=17), 6 \mathrm{~Hz}(\mathrm{n}=6)$ and $8 \mathrm{~Hz}(\mathrm{n}=6)$, thereby encompassing the physiological range for the rat. The experimental period consisted of an imposed $40 \%$ variability over the fixed (control) period. Once again, over an identical time period there was an equal number of beats both in the control and experimental protocols. Beatto-beat variability protocols were both preceded and followed by fixed rate periods. The variables from the beat-tobeat variability protocols were measured right after assessment of the fixed-rate response at the same base frequency, allowing the assessment and potential correction of rundown of the preparation. If rundown exceeded $20 \%$ per 5 consecutive protocols, the trabeculae were discarded. As a surrogate for standardized measurements of heart rate variability in the time domain, we calculated the effective SDNN (standard deviation of normal to normal intervals) spanning each of the individual 2 minute variable periods at each base frequency. These were then averaged and labeled as $\mathrm{SDNN}_{(\bmod )}$ index.

In a subsequent set of experiments $(n=3)$, intracellular calcium transients were measured after iontophoretic loading of the calcium indicator bis-Fura- 2 into a preparation. The dye loading technique and protocol that allows for assessment of calcium transients at body temperature has been described previously $[8,10]$. Briefly, at room temperature, a micropipette filled with bis-Fura- 2 is introduced into a single myocyte near the center of the preparation, and a small negative current is applied to introduce the dye into the cytoplasm. Via gap-junctions this dye spreads to neighboring cells. Once the dye has uniformly spread (on average 30 minutes), fluorescence intensity at $510 \mathrm{~nm}$ is measured during alternate exposure to 340 and $380 \mathrm{~nm}$ excitation light. Trabeculae were then stimulated with the previously described variable pacing protocols, Simultaneous force development and intracellular calcium transients were recorded at $37^{\circ} \mathrm{C}$.

Data was collected via custom-written Labview ${ }^{\circledR}$ programs, and stored for off-line analysis. Results are denoted as mean \pm SEM unless stated otherwise. Wherever applicable, paired or un-paired t-tests were performed, and a twotailed value of $\mathrm{P}<0.05$ was taken as level for significance.

\section{RESULTS}

In Fig. (1) we demonstrate that when pre-beat duration increased, so did contractile strength. In the depicted example, at a frequency of $8 \mathrm{~Hz}$ (cycle time of $125 \mathrm{~ms}$ ), a $40 \%$ variation was imposed, making the shortest beat-to-beat interval $100 \mathrm{~ms}$, and the longest interval $150 \mathrm{~ms}$. The highest contractile force was observed after the longest pre-beat duration (i.e. after the longest diastolic interval), whereas the shortest intervals were followed by the weakest beats.

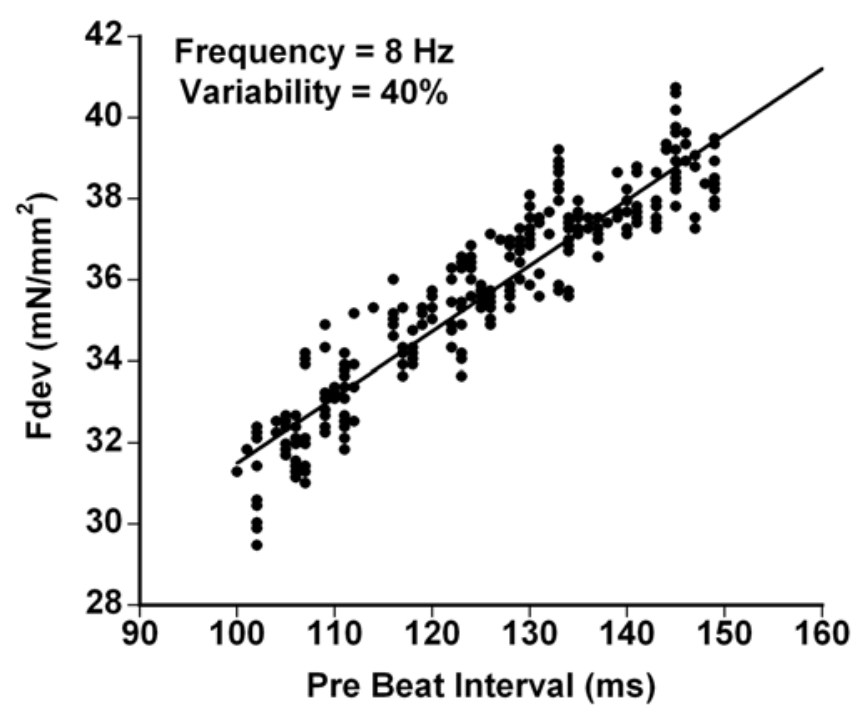

Fig. (1). Force development is dependent on duration of the prebeat interval; when previous beat-duration increases, so does peak twitch force development (Fdev). In the depicted example, at a frequency of $8 \mathrm{~Hz}$ a $40 \%$ variation was imposed. Thus, inter-beat duration varied from 100 to $150 \mathrm{~ms}$, with an average of $125 \mathrm{~ms}(=8$ $\mathrm{Hz})$.

For each stimulation period, we averaged the mean twitch force. By comparing the developed force at $4 \mathrm{~Hz}$ between fixed and variable pacing we noticed small, increases in force due to variable pacing with a trend towards increasing force with increasing varibility (with the notable excep- 
tion of $100 \%$ variability). The highest increase was noted at $120 \%$ variability $(+1.3 \%$, SEM $1.0 \mathrm{n}=17$, Fig. 2) although this did not achieve statistical significance.

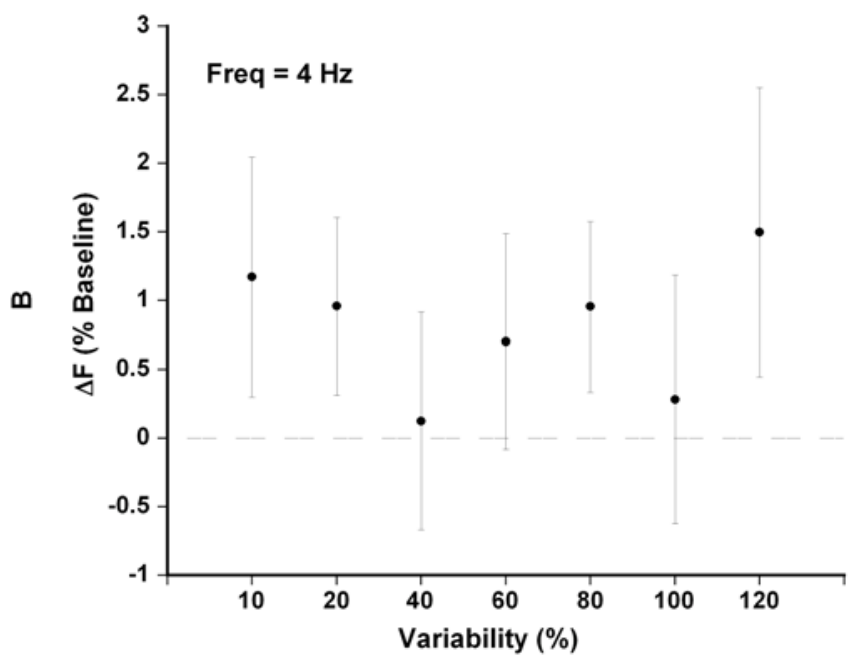

Fig. (2). At a base cycle time of $250 \mathrm{~ms}(4 \mathrm{~Hz})$, between 10 and $120 \%$ variability (reflecting cycle times from $237.5-262.5 \mathrm{~ms}$ and 125-375 ms resp.), the small increases in average Fdev were not significant, but on the other hand did not impair contractility either $(\mathrm{n}=17)$.

The $40 \%$ variability index imposed during these protocols is well within the normal physiologic intervals reported in the literature for rats whereas the $120 \%$ variability index exceeds the variations reported for "in vivo" experiments. [11] (Fig. 3).

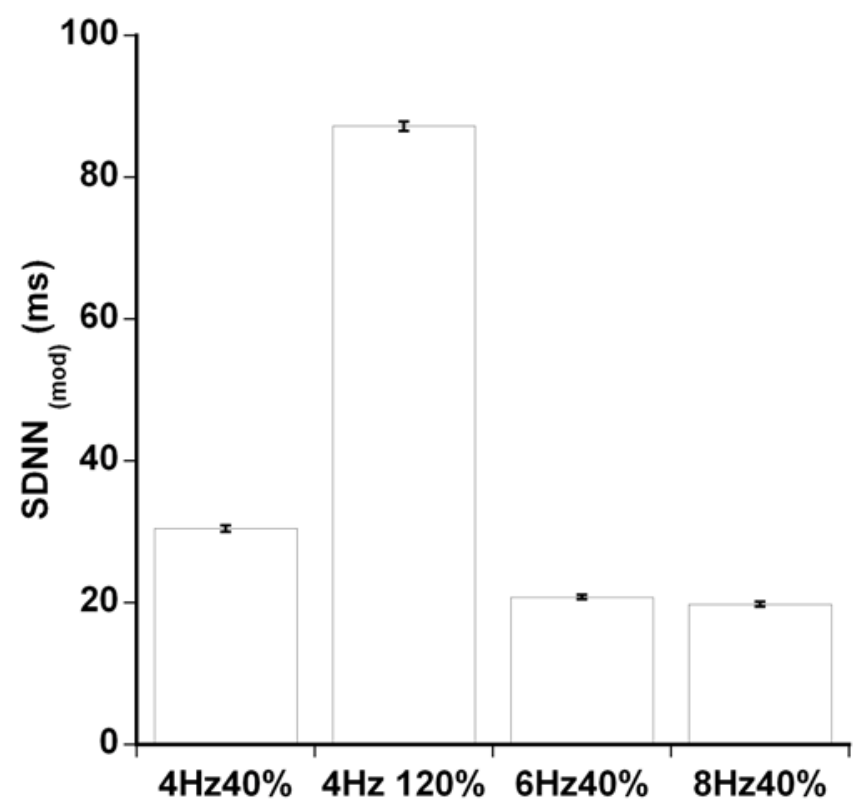

Fig. (3). At a variability of $40 \%$ and for all frequencies observed $(4,6,8)$ the $\mathrm{SDNN}_{\text {(mod) }}$ was well within the physiologic range for rats. At $120 \%$ variability the $\mathrm{SDNN}_{(\text {mod) }}$ was elevated.

The second experimental protocol group had baseline frequencies set at 4,6 and $8 \mathrm{~Hz}$ during the fixed rate intervals with the corresponding $40 \%$ variability algorithm imposed during alternate 2 minute periods.
Trabeculae, while exposed to variable beat-to-beat stimulation protocol, showed a more substantial increase in Fdev from an average of 15.1 to $16.5 \mathrm{mN} / \mathrm{mm}^{2}$, an average percentile increase of $10.1 \%(\mathrm{P}<0.05, \mathrm{n}=7, \mathrm{SEM} 1.9)$ at $8 \mathrm{~Hz}$ (Fig. 4) when compared to their fixed-rate stimulation averages. This indicates that despite the fact that the number of beats per minute was the same, beat-to-beat variability increased contractility.

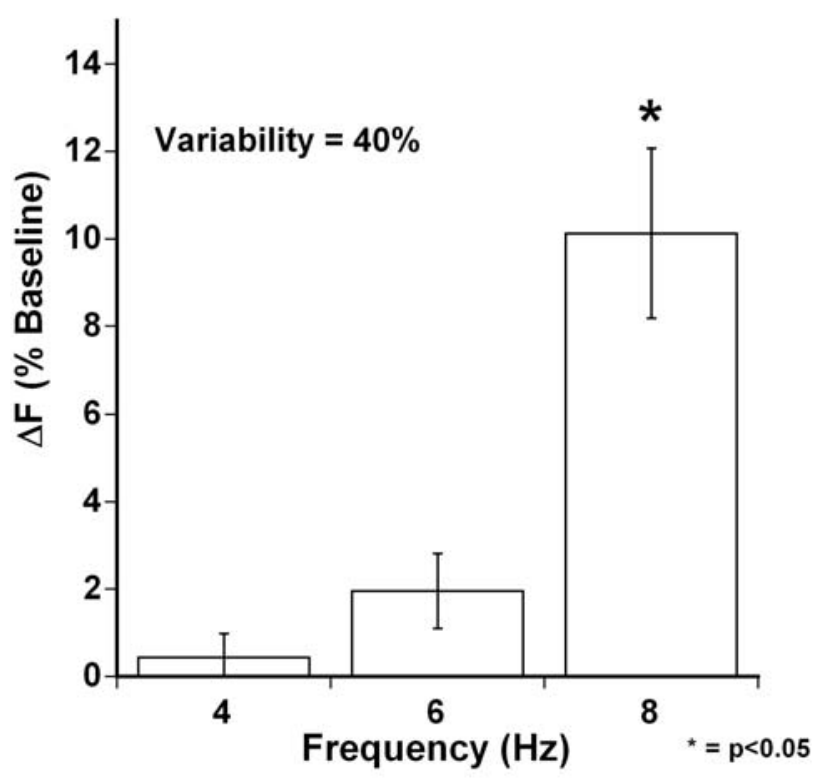

Fig. (4). At a variability of $40 \%$, average active developed force again was not significantly increased at an average cycle time of 4 and $6 \mathrm{~Hz}$ however at the $8 \mathrm{~Hz}$, the $40 \%$ variability significantly increased the average developed force of the trabeculae, $*(\mathrm{P}<0.05$, $\mathrm{n}=7$ ).

When beat-to-beat variability is imposed on cardiac trabeculae the effect of these interval dependent changes on the calcium transients can be seen in Fig. (5). In Fig. (5A), a positive correlation between the developed calcium transient amplitude and force of contraction (Fdev) was observed $(\mathrm{P}<0.05)$ under all tested conditions. In Fig. $(\mathbf{5 B})$ we demonstrate the positive correlation between a longer cycle length (pre-beat duration) with an increase in the corresponding calcium transient. In addition in Fig. (5C) the variations in interbeat duration and their associated fluctuations in the amplitude of the calcium transients are depicted.

When an inter-beat period exceeds the fixed-rate, the following stimulation results in an increase in amplitude of contracture. A beat that is shorter than the fixed-rate generally results in a decrease of contraction. However, as was the case for data obtained at a variability of $40 \%$ at 6 and $8 \mathrm{~Hz}$, these deviations from the fixed-rate average are not necessarily of identical magnitude. Thus, the absolute extra strength generated by long beats appears to be greater than the absolute loss by an equivalently shorter beat with corresponding changes in the calcium transients. On average, the introduction of variability can result in amplitudes of contraction during variable pacing that, on average, exceed the amplitude during the fixed-rate pacing protocol.

\section{DISCUSSION}

We set out to observe whether a small variation in the inter-beat period (beat-to-beat variability) has a modulating 

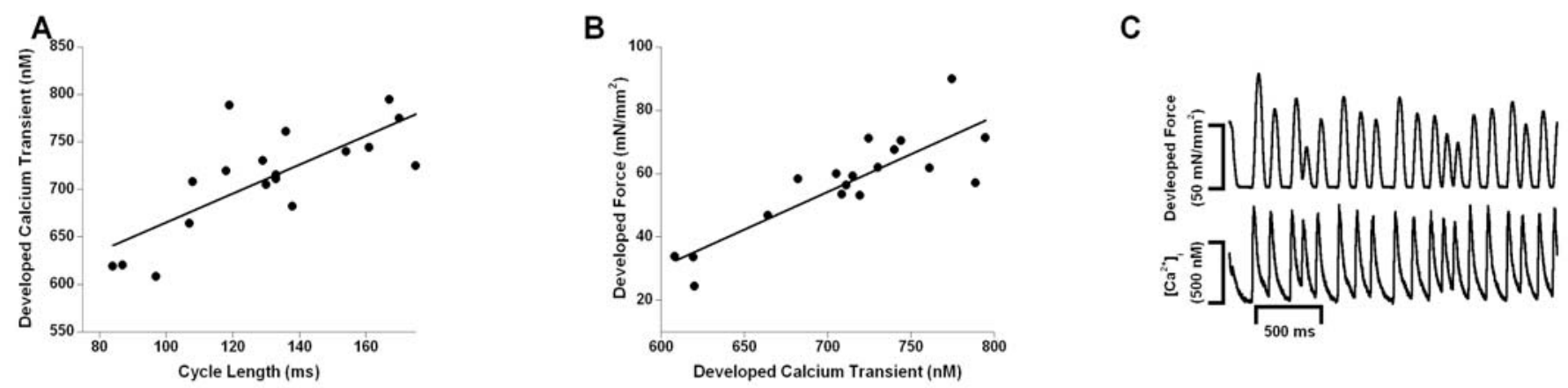

Fig. (5). At an average cycle rate of $125 \mathrm{~ms}(8 \mathrm{~Hz})$, a variation in inter-beat duration of $40 \%$ was applied. The correlation between the developed calcium transient and peak active force development ( Fdev) is shown in panel $\mathbf{A}\left(\mathrm{R}^{2}=0.57, \mathrm{p}=0.03\right)$. Panel $\mathbf{B}$ demonstrates the positive correlation between the variations in cycle length and the developed calcium transients $\left(\mathrm{R}^{2}=0.72, \mathrm{p}=0.0006\right)$. In panel $\mathbf{C}$, the top and bottom panel show the resulting intracellular calcium transients and twitch contractions respectively. The variation in inter-beat duration is associated with variations in the calcium transients.

effect on cardiac contractility. We showed that a nonvariable pacing algorithm can indeed alter myocardial contractile strength, and thus we demonstrate for the first time that beat-to-beat variability is a direct and positive modulator of cardiac contractility. Results from isolated muscle preparations including those regarding the intervalforce relationship have been well substantiated by other investigators utilizing different models [12]; the multicellular preparation has been validated and accepted to reflect most properties of the whole heart $[13,14]$. Our data therefore clearly suggests that under some of the conditions studied, beat-to-beat variability can actively contribute to cardiac contractile strength. For this proof of principle investigation, our focus was on the effects seen on developed force. Changes in diastolic force were not addressed and should be subjected to further investigation.

Post-rest potentiation [4, 15] experiments have shown that when stimulation is interrupted and re-established, the contractile strength of the first beat after the period of nonstimulation greatly depends on the duration of rest. This is very similar to what we observed, albeit in a much smaller time domain. Where post-rest experiments typically are assessed from 1 to 120 seconds, we observed a similar behavior for the short periods of rest (in case of variable beat duration exceeding the average heart-rate), or lack of rest (in case of beat duration shorter than the average heart-rate), within the sub-second/millisecond domains. Thus, although our results were obtained in a much smaller temporal domain, they are in line with the post-rest potentiation observations.

The molecular regulation of the phenomenon of positive inotropic beat-to-beat variability has to be sought in the regulation of calcium handling. The observed beat-to-beat variation in force correlated very closely with the calcium transient amplitude. This was expected, as regulation of calcium handling is at the basis of post-rest potentiation. Postrest potentiation occurs by $\mathrm{Ca}^{2+}$ loading of the SR during the rest interval. At the same time, at the level of the $\mathrm{Na}^{+} / \mathrm{Ca}^{2+}$ exchanger (NCX) an increase in intracellular calcium will lead to increase in activity which can decrease the intracytosolic calcium. Since both the function of the SR calcium ATPase and the NCX are influenced by many factors, including phosphorylation potential, transmembrane sodium gradient, and the cytosolic calcium level the competition between the sarcoplasmic reticulum (SR) calcium ATPase and the $\mathrm{Na}^{+} / \mathrm{Ca}^{2+}$ exchanger (NCX) all contribute to the development of the subsequent calcium transient. Several of these factors in turn change within the time-domain of interest. Thus, the resulting SR calcium load highly depends on many, rapidly changing, factors, and it is thus likely that there is not a simple linear relationship between SR calcium load and inter-beat duration. We speculate that a variable inter-beat duration therefore likely causes a small positive effect on the SR Calcium ATPase, this allows for more calcium to be available for s subsequent transient/beat resulting in the elevated average calcium transient. Moreover, at different heart rates, the above factors that modulate SR calcium ATPase and NCX activity are affected differently, impacting on the effect of beat-to-beat variability on contractility, as observed with a gradual effect of variability over the measured frequency range. It is thought that this differential effect on the modulating factors is highly responsible for the differences found between $4 \mathrm{~Hz}, 6 \mathrm{~Hz}$ and $8 \mathrm{HZ}$

Calcium is one of the most prominent signaling ions, and it is likely that an introduction of beat-to-beat variability that changes the intracellular calcium homeostasis may have secondary effects. Through various signaling mechanisms and resulting changes in protein expression, introduction of beatto-beat variability may have secondary effect, but to determine these were well beyond the scope of this proof-ofprinciple investigation.

In vivo, beat-to-beat variation occurs naturally, and has been termed heart rate variability (HRV). Although the underlying principle of our studies may be similar to those underlying HRV, we cannot unambiguously extrapolate our results to the in vivo situation, and whether introduction of beat-to-beat variation will have a beneficial effect in vivo in humans cannot be answered with this proof-of-principle investigation. Still, HRV is known to correlate with cardiac dysfunction. When humans age HRV becomes depressed [16]. A similar reduction in HRV is observed in patients suffering from end-stage cardiac failure [17-19]. If this reduction in HRV can be altered by introduction of an increase in the interbeat variability, this may potentially offer a novel therapeutic corridor. While there have been reports that irregular pacing may potentially worsen cardiac function in some patients [20-22] no direct measure of contractility was 
obtained. It remains unclear whether these results were in fact due to a decrease in contractility or to other associated factors such as inter and intraventricular dyssynchrony due to RV pacing, variable ventricular filling, and resulting loading conditions or other systemic effects. Thus, although it remains unresolved whether beat-to-beat variation could have similar effects in vivo. We show that under near physiological conditions and in a multicellular preparation that preserves most of the fundamental components of the myocardium, beat-to-beat variations can introduce an inotropic effect in vitro with corresponding changes in the measured calcium transients.

\section{CONCLUSION}

In conclusion, our data suggest that interbeat duration can, under certain conditions, be a determinant and modulator of cardiac contractility.

\section{REFERENCES}

[1] Hasenfuss G, Holubarsch C, Hermann HP, Astheimer K, Pieske B, Just $\mathrm{H}$. Influence of the force-frequency relationship on haemodynamics and left ventricular function in patients with non-failing hearts and in patients with dilated cardiomyopathy. Eur Heart $\mathbf{J}$ 1994; 15: 164-70.

[2] Layland J, Kentish JC. Positive force- and $\left[\mathrm{Ca}^{2+}\right] \mathrm{i}$-frequency relationships in rat ventricular trabeculae at physiological frequencies. Am J Physiol Heart Circ Physiol 1999; 276: H9-H18.

[3] Narayan P, McCune SA, Robitaille PM, Hohl CM, Altschuld RA. Mechanical alternans and the force-frequency relationship in failing rat hearts. J Mol Cell Cardiol 1995; 27: 523-30.

[4] Pieske B, Sutterlin M, Schmidt-Schweda S, et al. Diminished postrest potentiation of contractile force in human dilated cardiomyopathy. Functional evidence for alterations in intracellular $\mathrm{Ca}^{2+}$ handling. J Clin Invest 1996; 98: 764-76.

[5] Mulieri LA, Hasenfuss G, Ittleman F, Blanchard EM, Alpert NR. Protection of human left ventricular myocardium from cutting injury with 2,3-butanedione monoxime. Circ Res 1989; 65: 1441-9.

[6] Stuyvers BD, McCulloch AD, Guo J, Duff HJ, ter Keurs HE. Effect of stimulation rate, sarcomere length and $\mathrm{Ca}^{(2+)}$ on force generation by mouse cardiac muscle. J Physiol 2002; 544: 817-30.

[7] Raman S, Kelley MA, Janssen PM. Effect of muscle dimensions on trabecular contractile performance under physiological conditions. Pflugers Arch 2006; 451: 625-30.

[8] Janssen PML, Stull LB, Marban E. Myofilament properties comprise the rate-limiting step for cardiac relaxation at body temperature in the rat. Am J Physiol Heart Circ Physiol 2002; 282: H499H507.
[9] Rodriguez EK, Hunter WC, Royce MJ, Leppo MK, Douglas AS, Weisman HF. A method to reconstruct myocardial sarcomere lengths and orientations at transmural sites in beating canine hearts. Am J Physiol 1992; 263: H293-306.

[10] Stull LB, Leppo M, Marban E, Janssen PML. Physiological determinants of contractile force generation and calcium handling in mouse myocardium. J Mol Cell Cardiol 2002; 34: 1367-1376.

[11] Schaan BD, Dall'Ago P, Maeda CY, et al. Relationship between cardiovascular dysfunction and hyperglycemia in streptozotocininduced diabetes in rats. Braz J Med Biol Res 2004; 37: 1895-902.

[12] Zaugg CE, Kojima S, Wu ST, Wikman-Coffelt J, Parmley WW, Buser PT. Intracellular calcium transients underlying interval-force relationship in whole rat hearts: effects of calcium antagonists. Cardiovasc Res 1995; 30: 212-21.

[13] de Tombe PP, Little WC. Inotropic effects of ejection are myocardial properties. Am J Physiol Heart Circ Physiol 1994; 266: H1202-13.

[14] Sys SU, De Keulenaer GW, Brutsaert DL. Reappraisal of the multicellular preparation for the in vitro physiopharmacological evaluation of myocardial performance. Adv Exp Med Biol 1998; 453: 441-50; discussion 451.

[15] Janssen PML, Lehnart SE, Prestle J, Hasenfuss G. Preservation of contractile characteristics of human myocardium in multi-day cell culture. J Mol Cell Cardiol 1999; 31: 1419-27.

[16] Corino VD, Matteucci M, Mainardi LT. Analysis of heart rate variability to predict patient age in a healthy population. Methods Inf Med 2007; 46: 191-5.

[17] Panina G, Khot UN, Nunziata E, Cody RJ, Binkley PF. Assessment of autonomic tone over a 24-hour period in patients with congestive heart failure: relation between mean heart rate and measures of heart rate variability. Am Heart J 1995; 129: 748-53.

[18] Counihan PJ, Fei L, Bashir Y, Farrell TG, Haywood GA, McKenna WJ. Assessment of heart rate variability in hypertrophic cardiomyopathy. Association with clinical and prognostic features. Circulation 1993; 88: 1682-90.

[19] Saul JP, Arai Y, Berger RD, Lilly LS, Colucci WS, Cohen RJ. Assessment of autonomic regulation in chronic congestive heart failure by heart rate spectral analysis. Am J Cardiol 1988; 61: 1292-9.

[20] Daoud EG, Weiss R, Bahu M, et al. Effect of an irregular ventricular rhythm on cardiac output. Am J Cardiol 1996; 78: 1433-1436.

[21] Clark DM, Plumb VJ, Epstein AE, Kay GN. Hemodynamic effects of an irregular sequence of ventricular cycle lengths during atrial fibrillation. J Am Coll Cardiol 1997; 30: 1039-1045.

[22] Melenovsky V, Hay I, Fetics BJ, et al. Functional impact of rate irregularity in patients with heart failure and atrial fibrillation receiving cardiac resynchronization therapy. Eur Heart J 2005; 26: 705-711. 